\title{
A Novel Analytical Method for Maximum Likelihood Detection in MIMO Multiplexing Systems
}

\author{
Wei Peng, Shaodan Ma, Tung-sang Ng, Fellow, IEEE, and Jiangzhou Wang, Senior Member, IEEE
}

\begin{abstract}
This letter addresses the problem of symbol error probability (SEP) analysis for maximum likelihood (ML) detection in multiple-input multiple-output (MIMO) multiplexing systems. A new analytical method is presented based on the Total Probability Theorem. The effects of imperfect channel estimation and power allocation scheme are investigated. The accuracy of the proposed method is demonstrated by MonteCarlo simulations. It is shown that the analytical results match quite well with the simulation ones irrespective of the signal-tonoise-ratio (SNR).
\end{abstract}

Index Terms-MIMO, maximum likelihood detection, performance analysis, imperfect channel estimation.

\section{INTRODUCTION}

$\mathbf{I}^{\mathrm{N}}$ $\mathrm{N}$ recent years, multiple-input multiple-output (MIMO) technique has emerged as one of the most significant breakthroughs to increase system capacity without extra bandwidth. Generally, MIMO can be applied in two situations. One is in space-time coding systems where the transmission quality is improved due to spatial diversity. The other is in spatial multiplexing systems where independent signals are transmitted over different antennas to increase the transmission rate [1]. In the paper, the latter situation is considered.

The issue of signal detection for MIMO multiplexing systems has been widely discussed and a number of detection methods have been proposed, e.g., zero forcing (ZF), minimum mean square estimation (MMSE), vertical Bell Labs space time (V-BLAST) and maximum likelihood (ML) methods [2]. Among them, the ML detection is the optimal one from the error probability point of view. Since it is a non-linear detection using a full search process, performance analysis which is instructive for system designers is not straightforward and has attracted a lot of attentions recently. In the literature, the symbol error probability (SEP) or bit error probability (BEP) for each transmitted signal is generally written as the sum of weighted pair-wise error probabilities (PEP) where PEP means the probability that the receiver decides in favor of one signal vector when another signal vector is transmitted. Upper-bound/approximation of SEP is then derived based on the PEP calculation/approximation [3-8]. Unfortunately, all of these analytical bounds/approximations are tight only under high signal-to-noise-ratio (SNR) and there is a significant

Paper approved by P. Y. Kam, the Editor for Modulation and Detection of the IEEE Communications Society. Manuscript received August 30, 2007; revised June 4, 2008.

W. Peng was with the Department of Electrical and Electronic Engineering, University of Hong Kong. She is now with the Department of Electrical and Communication of Tohoku University, Japan (e-mail: weipeng@eee.hku.hk).

S. Ma and T.-s. Ng are with the Department of Electrical and Electronic Engineering, University of Hong Kong (e-mail: \{sdma, tsng\}@eee.hku.hk).

J. Wang is with the Department of Electronics, Kent University, UK (email: J.Z.Wang@kent.ac.uk).

Digital Object Identifier 10.1109/TCOMM.2009.08.70432 gap between the analytical and simulation results when the SNR is low. In addition, the existing analytical methods were developed under the assumption of equal power allocation on the transmit antennas. Their extension to systems with unequal power allocation which is generally the case in many practical systems, e.g., beamforming systems, is by no means straight forward.

In this letter, a new method to SEP analysis for the ML detection in a MIMO multiplexing system is proposed. In this method, the SEP for each transmitted signal is first expressed in terms of the SEPs conditioned on a set of error events corresponding to the other transmitted signals and the probabilities of these error events. By analyzing the post-detection SNR, the conditional SEPs are derived in closed-form and the average SEPs are finally obtained by solving a set of equations. The advantage of the proposed method is that imperfect channel estimation as well as unequal power allocation among the transmitted signals can also be considered, thus rendering the proposed method more practical. The accuracy of this SEP analysis is demonstrated by Monte-Carlo simulations. The comparison between the analytical and simulation results shows that they match quite well irrespective of the SNR. This provides system designers an effective way to predict more accurately the system performance before implementation, even under low SNR situation.

\section{System Model And ML Detection}

\section{A. System Model with Channel Estimation}

Consider a MIMO multiplexing system with $N_{t}$ transmit and $N_{r}$ receive antennas $\left(N_{r} \geq N_{t}\right)$. The baseband received signal vector is given by

$$
\mathbf{y}=\mathbf{H x}+\mathbf{n},
$$

where $\mathbf{y}$ is an $N_{r} \times 1$ received signal vector; $\mathbf{n}$ represents an $N_{r} \times 1$ additive complex Gaussian noise (AWGN) vector, each element being independent with zero mean and variance $\sigma_{n}^{2}$; $\mathbf{H}$ denotes an $N_{r} \times N_{t}$ channel matrix whose elements being independent and identically distributed (i.i.d) complex Gaussian variables with zero mean and unit variance $\left(\sigma_{h}^{2}=1\right)$; $\mathbf{x}$ is the $N_{t} \times 1$ transmitted signal vector with mutually independent signals. Let $C$ represent the constellation of the modulation. It is assumed that all the constellation symbols have equal probability. To simplify the derivation, Q-ary phase shift keying (QPSK) modulation is assumed. The proposed method can be easily extended to the system using other modulation schemes.

Denoting $\hat{\mathbf{H}}$ to be the estimated channel matrix, it is generally assumed that the elements of $\hat{\mathbf{H}}$ are i.i.d complex Gaussian variables with zero mean and variance $\sigma_{\hat{h}}^{2}$. It follows 
from [9] that the channel matrix $\mathbf{H}$ can be written as

$$
\mathbf{H}=\rho_{h, \hat{h}} \hat{\mathbf{H}}+\Delta \mathbf{H},
$$

where $\rho_{h, \hat{h}}=c_{h, \hat{h}} / \sigma_{\hat{h}}, c_{h, \hat{h}}$ is the correlation coefficient between the corresponding elements of $\hat{\mathbf{H}}$ and $\mathbf{H}, 0<c_{h, \hat{h}} \leq 1$; $\triangle \mathbf{H}$ represents the channel estimation error matrix whose $(j, i)^{\text {th }}$ element equals to $\triangle h_{j, i}=h_{j, i}-\rho_{h, \hat{h}} \hat{h}_{j, i}$ and can be modeled as an i.i.d complex Gaussian variable with zero mean and variance $\sigma_{\triangle h}^{2}=1-c_{h, \hat{h}}^{2}$ [9]. When perfect channel estimation is assumed, $\rho_{h, \hat{h}}=c_{h, \hat{h}}=1$. It should be noted that $\rho_{h, \hat{h}}$ and $c_{h, \hat{h}}$ are determined by the channel estimator. They vary with the SNR and both approach one when SNR increases. For any given SNR, $\rho_{h, \hat{h}}$ and $c_{h, \hat{h}}$ can be obtained by the method in [9] and are thus generally assumed to be known at the receiver [6-8].

Substituting (2) into (1), it yields

$$
\mathbf{y}=\left(\rho_{h, \hat{h}} \hat{\mathbf{H}}+\Delta \mathbf{H}\right) \mathbf{x}+\mathbf{n}=\rho_{h, \hat{h}} \hat{\mathbf{H}} \mathbf{x}+\mathbf{u},
$$

where $\mathbf{u}=\triangle \mathbf{H} \mathbf{x}+\mathbf{n}$ represents the interference plus noise and can be regarded as the equivalent noise vector with the $j^{\text {th }}$ element given as $u_{j}=\sum_{i=1}^{N_{t}} \triangle h_{j, i} x_{i}+n_{j}$. Since $\triangle h_{j, i}$ and $n_{j}$ are independent zero mean complex Gaussian variables with variance $\sigma_{\triangle h}^{2}$ and $\sigma_{n}^{2}$ respectively, it follows that for given $\mathbf{x}, u_{j}$ is also zero mean Gaussian variable [10] and the variance of $u_{j}$ is given by $\sigma_{u}^{2}=\operatorname{var}\left\{\sum_{i=1}^{N_{t}} \triangle h_{j, i} x_{i}+n_{j}\right\}=$ $E\left\{\left|\sum_{i=1}^{N_{t}} \triangle h_{j, i} x_{i}+n_{j}\right|^{2}\right\}=E_{s}\left(1-c_{h, \hat{h}}^{2}\right)+\sigma_{n}^{2}$ where $E_{s}=$ $\sum_{i=1}^{N_{t}}\left|x_{i}\right|^{2}$ denotes the total transmit power at the transmitter and $E\{\cdot\}$ represents expectation operation.

\section{B. Maximum Likelihood Detection}

When Gaussian noise is assumed, the ML detection for the transmitted signals can be realized as

$$
\tilde{\mathbf{x}}=\underset{\tilde{\mathbf{x}}}{\arg \min } \sum_{j=1}^{N_{r}}\left|y_{j}-\sum_{i=1}^{N_{t}} \rho_{h, \hat{h}} \hat{h}_{j, i} \tilde{x}_{i}\right|^{2},
$$

where $\tilde{\mathbf{x}}$ represents the decision vector for $\mathbf{x}$ and $\tilde{x}_{i}$ represents the decision for the $i^{\text {th }}$ transmitted signal. Due to discrete constellation points of the modulation, this is a non-linear detection and generally achieved by a full search process.

\section{Symbol ERror Probability}

Let $x_{i}$ be the $i^{\text {th }}\left(1 \leq i \leq N_{t}\right)$ transmitted signal and $X_{\bar{i}}=\left\{x_{1}, \ldots, x_{i-1}, x_{i+1}, \ldots, x_{N_{t}}\right\}$ be the other transmitted signals. The SEP of $x_{i}$ can be written in terms of the SEPs conditioned on a set of error events corresponding to $X_{\bar{i}}$ and the probabilities of these error events as

$$
\begin{aligned}
& P\left(\tilde{x}_{i} \neq x_{i}\right) \\
= & P\left(\tilde{x}_{i} \neq x_{i} \mid \operatorname{err}(\emptyset)\right) P(\operatorname{err}(\emptyset))+ \\
& \sum_{k=1, k \neq i}^{N_{t}} P\left(\tilde{x}_{i} \neq x_{i} \mid \operatorname{err}(k)\right) P(\operatorname{err}(k))+ \\
& \sum_{l=1, l \neq i}^{N_{t}} \sum_{m=l+1, m \neq i}^{N_{t}} P\left(\tilde{x}_{i} \neq x_{i} \mid \operatorname{err}(l, m)\right) \\
& \cdot P(\operatorname{err}(l, m))+\ldots+P\left(\tilde{x}_{i} \neq x_{i} \mid \operatorname{err}(\Omega)\right) P(\operatorname{err}(\Omega)),
\end{aligned}
$$

where $\operatorname{err} \emptyset), \operatorname{err}(k), \operatorname{err}(l, m)$ and $\operatorname{err}(\Omega)$ respectively represent no error, one error $\left(\tilde{x}_{k} \neq x_{k}\right)$, two errors $\left(\tilde{x}_{l} \neq x_{l}\right.$, $\left.\tilde{x}_{m} \neq x_{m}\right)$ and all errors in $X_{\bar{i}}$. Let $\operatorname{err}(\cdot)$ represent an error event of $X_{\bar{i}}, P\left(\tilde{x}_{i} \neq x_{i} \mid \operatorname{err}(\cdot)\right)$ denotes the SEP of $x_{i}$ conditioned on $\operatorname{err}(\cdot)$ and $P(\operatorname{err}(\cdot))$ stands for the probability of $\operatorname{err}(\cdot)$. Equation (5) is also known as the Total Probability Theorem. It is obvious that if each pair of $P\left(\tilde{x}_{i} \neq x_{i} \mid \operatorname{err}(\cdot)\right)$ and $P(\operatorname{err}(\cdot))$ in (5) is known, the SEP $P\left(\tilde{x}_{i} \neq x_{i}\right)$ can be easily calculated. Note that this SEP expression is universally applicable to arbitrary MIMO systems.

\section{A. The SEP Conditioned on $\operatorname{err}(\emptyset): P\left(\tilde{x}_{i} \neq x_{i} \mid \operatorname{err}(\emptyset)\right)$}

The SEP conditioned on the event $\operatorname{err}(\emptyset)$ is analyzed first. The result in this sub-section will form the basis for the following analysis on the SEPs conditioned on the other error events. Under the event $\operatorname{err}(\emptyset)$, no error occurs to the other transmitted signals. It means that $\tilde{x}_{q}=x_{q}\left(q=1, i-1, i+1, \ldots, N_{t}\right)$. From (3) and (4), the detection of $x_{i}$ becomes

$$
\tilde{x}_{i}=\underset{\tilde{x}_{i}}{\arg \min } \sum_{j=1}^{N_{r}}\left|\rho_{h, \hat{h}} \hat{h}_{j, i} x_{i}+u_{j}-\rho_{h, \hat{h}} \hat{h}_{j, i} \tilde{x}_{i}\right|^{2} .
$$

In fact, the detection of $x_{i}$ in (6) is equivalent to the ML detection in a single input multiple output (SIMO) system. Thus the result for a SIMO system in [11] can be applied here and the post-detection SNR is given as

$$
\gamma_{i, \operatorname{err}(\emptyset)}^{\mathrm{ML}}=\sum_{j=1}^{N_{r}} \rho_{h, \hat{h}}^{2}\left|\hat{h}_{j, i}\right|^{2}\left|x_{i}\right|^{2} / \sigma_{u}^{2}=c_{h, \hat{h}}^{2} \omega_{i}\left|x_{i}\right|^{2} / \sigma_{u}^{2},
$$

where $\omega_{i}=\sum_{j=1}^{N_{r}}\left|\hat{h}_{j, i}\right|^{2} / \sigma_{\hat{h}}^{2}$. It follows from [12] that for QPSK modulated systems, the SEP conditioned on $x_{i}, \omega_{i}$ and $\operatorname{err}(\emptyset)$ can be written as

$$
\begin{aligned}
& p\left(\tilde{x}_{i} \neq x_{i} \mid x_{i}, \omega_{i}, \operatorname{err}(\emptyset)\right) \\
& =G\left(\gamma_{i, \operatorname{err}(\emptyset)}^{\mathrm{ML}}\right) \\
& =2 Q\left(\sqrt{\gamma_{i, \operatorname{err}(\emptyset)}^{\mathrm{ML}}}\right)-Q^{2}\left(\sqrt{\gamma_{i, \operatorname{err}(\emptyset)}^{\mathrm{ML}}}\right) \\
& =\frac{1}{\pi} \int_{0}^{3 \pi / 4} \exp \left(\frac{-c_{h, \hat{h}}^{2} \omega_{i}\left|x_{i}\right|^{2}}{2 \sigma_{u}^{2} \sin ^{2} \theta}\right) d \theta .
\end{aligned}
$$

where $Q(t)=\int_{t}^{\infty} 1 / \sqrt{2 \pi} \cdot \exp \left(-z^{2} / 2\right) d z$. It should be noted that the function $G(\cdot)$ in (8) depends on the modulation scheme. It is straight-forward to apply this method to the system using other modulations by altering $G(\cdot)$. For example, for sixteen quadrature amplitude modulation (16QAM), $G(t)=1-[1-1.5 Q(\sqrt{0.2 t})]^{2}[10]$.

By averaging (8) with respect to the statistics of $x_{i}$ and $\omega_{i}$, the average conditional SEP can be achieved as

$$
\begin{aligned}
P & \left(\tilde{x}_{i} \neq x_{i} \mid \operatorname{err}(\emptyset)\right) \\
= & \frac{1}{\pi} \sum_{x_{i} \in C} p\left(x_{i}\right) \int_{0}^{\infty} p\left(\tilde{x}_{i} \neq x_{i} \mid x_{i}, \omega_{i}, \operatorname{err}(\emptyset)\right) p\left(\omega_{i}\right) d \omega_{i} \\
= & \frac{1}{\pi} \sum_{x_{i} \in C} p\left(x_{i}\right) \int_{0}^{\frac{3 \pi}{4}} \int_{0}^{\infty} \exp \left(-\frac{c_{h, h^{2}}^{2} \omega_{i}\left|x_{i}\right|^{2}}{2 \sigma_{u}^{2} \sin ^{2} \theta}\right) \\
& \cdot p\left(\omega_{i}\right) d\left(\omega_{i}\right) d \theta,
\end{aligned}
$$

where $p\left(x_{i}\right)$ and $p\left(\omega_{i}\right)$ are the probability density function (p.d.f) of $x_{i}$ and $\omega_{i}$, respectively. Apparently from the definition of $\omega_{i}$, it is a chi-square distributed variable [10] with $2 N_{r}$ degrees of freedom. It follows that the p.d.f of $\omega_{i}$ is given by

$$
p\left(\omega_{i}\right)=\omega_{i}^{N_{r}-1} \exp \left(-\omega_{i}\right) /\left(N_{r}-1\right) !
$$


Substituting (10) into (9), the conditional SEP becomes

$$
\begin{aligned}
P & \left(\tilde{x}_{i} \neq x_{i} \mid \operatorname{err}(\emptyset)\right) \\
= & \frac{1}{\pi} \sum_{x_{i} \in C} p\left(x_{i}\right) \int_{0}^{\frac{3 \pi}{4}}\left(1+\frac{c_{h, \hat{h}}^{2}\left|x_{i}\right|^{2}}{2 \sigma_{u}^{2} \sin ^{2} \theta}\right)^{-N_{r}} d \theta \\
= & \frac{1}{\pi} \sum_{x_{i} \in C} p\left(x_{i}\right)\left(\frac{3 \pi}{4}+\sum_{i=1}^{N_{r}}(-1)^{i} \sum_{j=0}^{i-1} \frac{a_{\operatorname{err}(\emptyset)}^{i-j-1 / 2}}{\left(1+a_{\operatorname{err}(\emptyset)}\right)^{i-1 / 2}}\right. \\
& \cdot\left(\begin{array}{c}
N_{r} \\
i
\end{array}\right) \cdot\left(\begin{array}{c}
i-1 \\
j
\end{array}\right) \cdot I\left(0,-\sqrt{\left.\left.\frac{1+a_{\operatorname{err}(\emptyset)}}{a_{\operatorname{err}(\emptyset)}},(j+1)\right)\right),}\right.
\end{aligned}
$$

where the parameter $a_{\operatorname{err}(\emptyset)}=c_{h, \hat{h}}^{2}\left|x_{i}\right|^{2} / 2 \sigma_{u}^{2}$. Note that $\int_{0}^{\infty} x^{n} \exp (-\mu x) d x=n ! \mu^{-n-1}$ [13] and the results in [14] are used in the above derivation. Function $I\left(t_{1}, t_{2}, n\right)$ is defined in [14] as

$$
\begin{aligned}
& I\left(t_{1}, t_{2}, n\right) \\
& =\frac{(2 n-3) ! !}{2^{n-1}(n-1) !}\left(\arctan \left(t_{2}\right)-\arctan \left(t_{1}\right)\right) \\
& \quad+\frac{(2 n-3) ! !}{(n-1) !} \sum_{k=1}^{n-1} \frac{(n-k-1) !}{2^{k}(2 n-2 k-1) ! !} \\
& \quad \cdot\left(\frac{t_{2}}{\left(1+t_{2}^{2}\right)^{n-k}}-\frac{t_{1}}{\left(1+t_{1}^{2}\right)^{n-k}}\right),
\end{aligned}
$$

where $(\cdot) ! !$ represents the double factorial and $\arctan (\cdot)$ represents the inverse tangent function.

\section{B. The SEPs Conditioned on the Other Error Events}

Under the event $\operatorname{err}(k), \tilde{x}_{k} \neq x_{k}(k \neq i)$ and $\tilde{x}_{q}=x_{q}(q=$ $\left.1, \cdots, i-1, i+1, \cdots, N_{t}, q \neq k\right)$. The received signal vector can be rewritten as

$$
\mathbf{y}=\sum_{i=1, i \neq k}^{N_{t}} \rho_{h, \hat{h}} \hat{\mathbf{h}}_{i} x_{i}+\rho_{h, \hat{h}} \hat{\mathbf{h}}_{k} \tilde{x}_{k}+\rho_{h, \hat{h}} \hat{\mathbf{h}}_{k} \Delta x_{k}+\mathbf{u},
$$

where $\hat{\mathbf{h}}_{i}$ is the $i^{\text {th }}$ column vector of $\hat{\mathbf{H}}$ and $\Delta x_{k}=x_{k}-\tilde{x}_{k}$. This situation can be regarded as if $\tilde{x}_{k}$ is transmitted from the $k^{\text {th }}$ antenna and $\rho_{h, \hat{h}} \hat{\mathbf{h}}_{k} \Delta x_{k}$ in (13) will then be treated as interference. As a result, $\rho_{h, h} \hat{\mathbf{h}}_{k} \Delta x_{k}+\mathbf{u}$ can also be considered as the equivalent noise vector and the received signal vector can be expressed as

$$
\mathbf{y}=\sum_{i=1, i \neq k}^{N_{t}} \rho_{h, \hat{h}} \hat{\mathbf{h}}_{i} x_{i}+\rho_{h, \hat{h}} \hat{\mathbf{h}}_{k} \tilde{x}_{k}+\mathbf{v}_{k},
$$

where $\mathbf{v}_{k}=\rho_{h, \hat{h}} \hat{\mathbf{h}}_{k} \Delta x_{k}+\mathbf{u}$ denotes the equivalent noise vector with its $j^{\text {th }}$ element given by $v_{k, j}=\rho_{h, h} \hat{h}_{j, k} \Delta x_{k}+$ $\sum_{i=1}^{N_{t}} \Delta h_{j, i} x_{i}+n_{j}$. Since $\hat{h}_{j, k}, \Delta h_{j, i}$ and $n_{j}$ are independent zero mean complex Gaussian variables with variance $\sigma_{\hat{h}}^{2}, \sigma_{\Delta h}^{2}$ and $\sigma_{n}^{2}$ respectively, it follows that for given $\mathbf{x}$ and $\Delta x_{k}, v_{k, j}$ is also complex Gaussian variable with zero mean and variance given by [10]

$$
\begin{aligned}
\sigma_{v, k}^{2} & =\operatorname{var}\left\{\rho_{h, \hat{h}} \hat{h}_{j, k} \Delta x_{k}+\sum_{i=1}^{N_{t}} \Delta h_{j, i} x_{i}+n_{j}\right\} \\
& =c_{h, \hat{h}}^{2}\left|\Delta x_{k}\right|^{2}+\sigma_{u}^{2} .
\end{aligned}
$$

As the symbol error occurs in adjacent positions in the constellation with the highest probability, it is reasonable to assume that error only happens between the transmitted symbol and its nearest constellation neighbor. Under this assumption, $\left|\Delta x_{k}\right|^{2}$ can be approximated by $\left|\Delta x_{k}\right|^{2} \approx$ $\min \left(d_{k, c}^{2}\right)=\alpha_{k} E\left\{\left|x_{k}\right|^{2}\right\}$, where $\min \left(d_{k, c}^{2}\right)$ represents the minimum squared Euclidean distance (SED) between $x_{k}$ and its constellation neighbors and $\alpha_{k}$ stands for the ratio of $\min \left(d_{k, c}^{2}\right)$ to the average transmit power of $x_{k}$. Note that $\min \left(d_{k, c}^{2}\right)$ and $\alpha_{k}$ vary with the modulation scheme. For QPSK modulation, $\min \left(d_{k, c}^{2}\right)=2 E\left\{\left|x_{k}\right|^{2}\right\}$ and $\alpha_{k}=2$.

Now the average SEP conditioned on $\operatorname{err}(k)$ can be analyzed in the same way as in sub-Section III-A. From (4) and (14), the detection of $x_{i}$ satisfies

$$
\tilde{x}_{i}=\underset{\tilde{x}_{i}}{\arg \min } \sum_{j=1}^{N_{r}}\left|\rho_{h, \hat{h}} \hat{h}_{j, i} x_{i}+v_{k, j}-\rho_{h, \hat{h}} \hat{h}_{j, i} \tilde{x}_{i}\right|^{2} .
$$

It follows that the post-detection SNR is $\gamma_{i, \operatorname{err}(k)}^{\mathrm{ML}}=$ $\sum_{j=1}^{N_{r}} \rho_{h, \hat{h}}^{2}\left|\hat{h}_{j, i}\right|^{2}\left|x_{i}\right|^{2} / \sigma_{v, k}^{2}=c_{h, \hat{h}}^{2} \omega_{i}\left|x_{i}\right|^{2} / \sigma_{v, k}^{2}$ and the conditional SEP $P\left(\tilde{x}_{i} \neq x_{i} \mid \operatorname{err}(k)\right)$ is given similarly to (9) and (11) as

$$
\begin{aligned}
P & \left(\tilde{x}_{i} \neq x_{i} \mid \operatorname{err}(k)\right) \\
= & \frac{1}{\pi} \sum_{x_{i} \in C} p\left(x_{i}\right)\left(\frac{3 \pi}{4}+\sum_{i=1}^{N_{r}}(-1)^{i} \sum_{j=0}^{i-1} \frac{a_{\mathrm{err}(k)}^{i-j-1 / 2}}{\left(1+a_{\mathrm{err}(k)}\right)^{i-1 / 2}}\right. \\
& \left.\cdot\left(\begin{array}{c}
N_{r} \\
i
\end{array}\right) \cdot\left(\begin{array}{c}
i-1 \\
j
\end{array}\right) \cdot I\left(0,-\sqrt{\frac{1+a_{\mathrm{err}(k)}}{a_{\mathrm{err}(k)}}},(j+1)\right)\right),
\end{aligned}
$$

where $a_{\operatorname{err}(k)}=c_{h, \hat{h}}^{2}\left|x_{i}\right|^{2} / 2 \sigma_{v, k}^{2}$. Similarly, the average SEP conditioned on other error events can also be derived straight forwardly as

$$
\begin{aligned}
P & \left(\tilde{x}_{i} \neq x_{i} \mid \operatorname{err}(l, m)\right) \\
= & \frac{1}{\pi} \sum_{x_{i} \in C} p\left(x_{i}\right)\left(\frac{3 \pi}{4}+\sum_{i=1}^{N_{r}}(-1)^{i} \sum_{j=0}^{i-1} \frac{a_{\operatorname{err}(l, m)}^{i-j-1 / 2}}{\left(1+a_{\operatorname{err}(l, m)}\right)}\right. \\
& \cdot\left(\begin{array}{c}
N_{r} \\
i
\end{array}\right) \cdot\left(\begin{array}{c}
i-1 / 2 \\
j
\end{array}\right) I\left(0,-\sqrt{\left.\left.\frac{1+a_{\operatorname{err}(l, m)}}{a_{\operatorname{err}(l, m)}},(j+1)\right)\right)}\right. \\
P & \left(\tilde{x}_{i} \neq x_{i} \mid \operatorname{err}(\Omega)\right) \\
= & \frac{1}{\pi} \sum_{x_{i} \in C} p\left(x_{i}\right)\left(\frac{3 \pi}{4}+\sum_{i=1}^{N_{r}}(-1)^{i} \sum_{j=0}^{i-1} \frac{a_{\operatorname{err}(\Omega)}^{i-j-1 / 2}}{\left(1+a_{\operatorname{err}(\Omega)}\right)}\right. \\
& \left.\cdot\left(\begin{array}{c}
N_{r} \\
i
\end{array}\right) \cdot\left(\begin{array}{c}
i-1 / 2 \\
j
\end{array}\right) I\left(0,-\sqrt{\frac{1+a_{\operatorname{err}(\Omega)}}{a_{\operatorname{err}(\Omega)}},(j+1)}\right)\right),
\end{aligned}
$$

where $a_{\operatorname{err}(l, m)}=c_{h, \hat{h}}^{2}\left|x_{i}\right|^{2} / 2 \sigma_{v, l, m}^{2}, \sigma_{v, l, m}^{2} \approx c_{h, \hat{h}}^{2}\left(\alpha_{l} E\{\mid\right.$ $\left.\left.\left.x_{l}\right|^{2}\right\}+\alpha_{m} E\left\{\left|x_{m}\right|^{2}\right\}\right)+\sigma_{u}^{2}, a_{\operatorname{err}(\Omega)}=c_{h, \hat{h}}^{2}\left|x_{i}\right|^{2} / 2 \sigma_{v, \Omega}^{2}$ and $\sigma_{v, \Omega}^{2} \approx c_{h, \hat{h}}^{2} \sum_{k=1, k \neq i}^{N_{t}} \alpha_{k} E\left\{\left|x_{k}\right|^{2}\right\}+\sigma_{u}^{2}$.

\section{The Probability of the Error Events $P(\operatorname{err}(\cdot))$ and the SEP Equations}

According to the probability theory, the probabilities $P(\operatorname{err}(\emptyset)), P(\operatorname{err}(k)), \cdots, P(\operatorname{err}(\Omega))$ can be expressed in 
terms of the SEPs $\left(P\left(\tilde{x}_{i} \neq x_{i}\right), i=1, \cdots, N_{t}\right)$ as

$$
\left\{\begin{array}{ll}
P(\operatorname{err}(\emptyset))= & \prod_{j=1, j \neq i}^{N_{t}}\left(1-P\left(\tilde{x}_{j} \neq x_{j}\right)\right) \\
P(\operatorname{err}(k))= & P\left(\tilde{x}_{k} \neq x_{k}\right) \\
& \cdot \prod_{j=1, j \neq i, k}^{N_{t}}\left(1-P\left(\tilde{x}_{j} \neq x_{j}\right)\right) \\
P(\operatorname{err}(l, m))= & P\left(\tilde{x}_{l} \neq x_{l}\right) P\left(\tilde{x}_{m} \neq x_{m}\right) \\
& \cdot \prod_{j=1, j \neq i, l, m}^{N_{t}}\left(1-P\left(\tilde{x}_{j} \neq x_{j}\right)\right) \\
\vdots & \\
P(\operatorname{err}(\Omega))= & \prod_{j=1, j \neq i}^{N_{t}} P\left(\tilde{x}_{j} \neq x_{j}\right)
\end{array} .\right.
$$

Applying the conditional SEPs ((11), (17) and (18)) and the probabilities of the error events (19) into (5), a set of SEP equations will be generated. Thus, the SEPs $P\left(\tilde{x}_{i} \neq x_{i}\right)(i=$ $\left.1, \cdots, N_{t}\right)$ can be obtained by solving the SEP equations. Generally, the power allocation will affect the SEPs. In the following, the SEP equations under equal and unequal power allocations will be illustrated in details. Note that the number of SEP equations depends on the power allocation scheme and it is always equal to the number of the unknown SEPs to be solved.

1) SEP Equation under Equal Power Allocation: When equal power is allocated among all the transmitted signals, the SEPs of each transmitted signal are the same, $P\left(\tilde{x}_{1} \neq\right.$ $\left.x_{1}\right)=\cdots=P\left(\tilde{x}_{N_{t}} \neq x_{N_{t}}\right)=e$. It follows from (5), (11), (17), (18) and (19) that the SEP equation is given by

$$
e=\sum_{j=0}^{N_{t}-1}\left(\begin{array}{c}
N_{t}-1 \\
j
\end{array}\right) \beta_{j} e^{j}(1-e)^{N_{t}-j-1}
$$

where $\beta_{0}=P\left(\tilde{x}_{i} \neq x_{i} \mid \operatorname{err}(\emptyset)\right)$ and $\beta_{j}=P\left(\tilde{x}_{i} \neq\right.$ $\left.x_{i} \mid \operatorname{err}\left(l_{1}, \cdots, l_{j}\right)\right),\left(l_{1}, \cdots, l_{j}\right)$ is a $j$-elements sub-set of $\left\{1, \cdots, i-1, i+1, \cdots, N_{t}\right\}$. The SEP $e$ can be obtained by solving (20).

2) SEP Equations under Unequal Power Allocation: For simplicity, it is assumed that the $q^{\text {th }}$ transmitted signal is allocated a different power from the others, while all the other transmitted signals have the same power. The SEP of $x_{q}$ is therefore different from that of the other transmitted signals. It yields

$$
P\left(\tilde{x}_{i} \neq x_{i}\right)= \begin{cases}\epsilon_{1}, & i \neq q \\ \epsilon_{2}, & i=q\end{cases}
$$

In this situation, two SEP equations are obtained from (5), (11), (17), (18) and (19) as:

$$
\left\{\begin{aligned}
\epsilon_{1}= & \eta_{0, \bar{q}}\left(1-\epsilon_{1}\right)^{N_{t}-2}\left(1-\epsilon_{2}\right) \\
& +\sum_{j=1}^{N_{t}-2}\left(\begin{array}{c}
N_{t}-2 \\
j-1
\end{array}\right) \eta_{j, q}\left(1-\epsilon_{1}\right)^{N_{t}-j-1} \epsilon_{1}^{j-1} \epsilon_{2} \\
& +\sum_{j=1}^{N_{t}-2}\left(\begin{array}{c}
N_{t}-2 \\
j
\end{array}\right) \eta_{j, \bar{q}}\left(1-\epsilon_{1}\right)^{N_{t}-j-2} \epsilon_{1}^{j}\left(1-\epsilon_{2}\right) \\
& +\eta_{N_{t}-1, q} \epsilon_{1}^{N_{t}-2} \epsilon_{2} \\
\epsilon_{2}= & \sum_{j=0}^{N_{t}-1}\left(\begin{array}{c}
N_{t}-1 \\
j
\end{array}\right) \delta_{j}\left(1-\epsilon_{1}\right)^{N_{t}-j-1} \epsilon_{1}^{j}
\end{aligned}\right.
$$

where

$$
\begin{gathered}
\begin{cases}\eta_{j, q}=P\left(\tilde{x}_{i} \neq x_{i} \mid \operatorname{err}\left(l_{1}, \cdots, l_{j}\right)\right), & i \neq q, j>0, \\
\eta_{j, \bar{q}}=P\left(\tilde{x}_{i} \neq x_{i} \mid \operatorname{err}\left(l_{1}, \cdots, l_{j}\right)\right), & i \neq q, j>0, \\
& q \notin\left\{l_{1}, \cdots, l_{j}\right\} \\
\eta_{0, \bar{q}}=P\left(\tilde{x}_{i} \neq x_{i} \mid \operatorname{err}(\emptyset)\right), & i \neq q\end{cases} \\
\begin{cases}\delta_{j}=P\left(\tilde{x}_{i} \neq x_{i} \mid \operatorname{err}\left(l_{1}, \cdots, l_{j}\right)\right), & i=q, j>0, \\
\delta_{0}=P\left(\tilde{x}_{i} \neq x_{i} \mid \operatorname{err}(\emptyset)\right), & q \notin\left\{l_{1}, \cdots, l_{j}\right\} .\end{cases}
\end{gathered}
$$

The SEPs $\epsilon_{1}, \epsilon_{2}$ can then be obtained by solving (22).

3) Discussion: When the number of transmit antennas is more than/equal to three, equations (20) or (22) may have multiple solutions. Since the SEPs should fall in the range of $[0,1]$, only the meaningful solution will be chosen.

For the situations when more than two levels of transmit powers are allocated among different transmit antennas or when the number of transmit antennas is more than three, the SEP equations will become more complicated. To obtain the solutions for such SEP equations, the assistance of computation tools, such as the toolbox "fsolve" in Matlab, may be needed.

Remarks: Unlike the existing methods where the upper bound/approximation of SEP is derived based on the PEP calculation/approximation, the proposed method directly develops the SEP based on the SEPs conditioned on a set of error events. Since the closed-form conditional SEPs as a function of SNR and modulation scheme are directly derived, it is expected that the proposed method will yield accurate solutions. This will be verified in the following section. On the other hand, due to the PEP approximation in the existing methods, there is generally a significant gap between the analytical and simulation results in the low SNR region.

\section{NumERicAl AND Simulation RESUlts}

A system with two transmit antennas is taken as an example. The number of receive antennas are set to be two and four, respectively. Two cases are considered: 1) the channel matrix is perfectly estimated (perfect CSI at the receiver); 2) the channel estimation matrix is obtained by least squares estimator [15] with the aid of pilot signals (imperfect channel estimation). The results are given with respect to SNR defined as $S N R=\left(E\left\{\|\mathbf{x}\|^{2}\right\} / N_{t}\right) /\left(E\left\{\|\mathbf{n}\|^{2}\right\} / N_{r}\right)$. The simulation results are obtained by averaging the results over $10^{6}$ Monte Carlo realizations.

Equal power allocation is considered first and unit transmit power is assumed for both of the transmit antennas ( $\left.E\left\{\left|x_{1}\right|^{2}\right\}=E\left\{\left|x_{2}\right|^{2}\right\}=1\right)$. In this situation, the average SEP of $x_{1}$ is the same as that of $x_{2}$ which is the solution of equation (20). The comparison between the analytical and simulation results is shown in Fig. 1 for the systems with $N_{r}=2$ and $N_{r}=4$. It is clear that the analytical results are very close to the simulation ones irrespective of channel estimation error or SNR. Next, unequal power allocation is considered and the transmit power of $x_{1}$ and $x_{2}$ are set to be $E\left\{\left|x_{1}\right|^{2}\right\}=7 / 4$ and $E\left\{\left|x_{2}\right|^{2}\right\}=1 / 4$, respectively. 


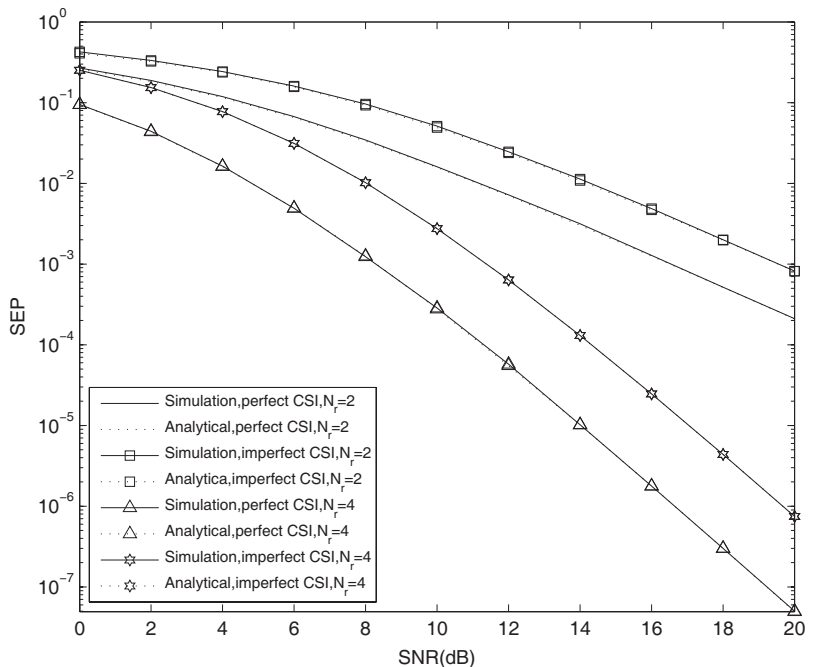

Fig. 1. SEP for a QPSK modulated system, $E\left\{\left|x_{1}\right|^{2}\right\}=E\left\{\left|x_{2}\right|^{2}\right\}=1$.

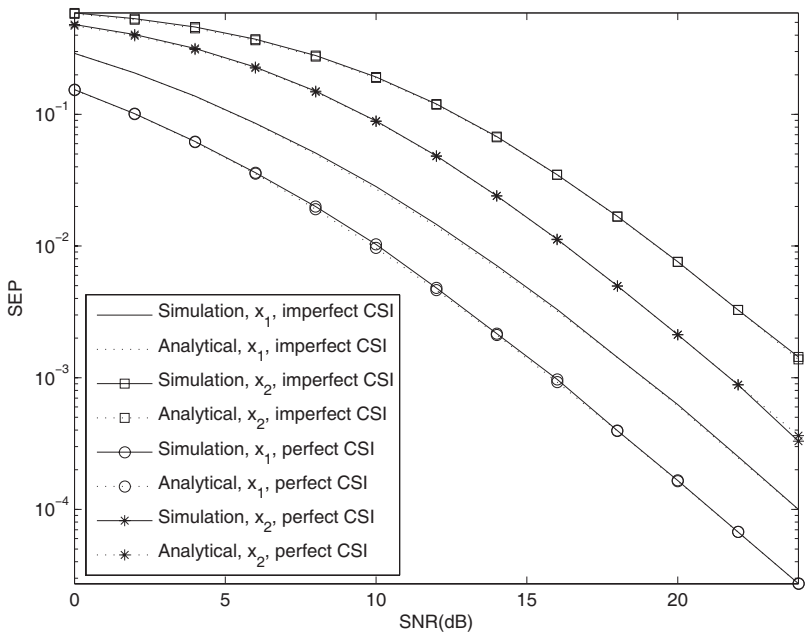

Fig. 2. SEP for a QPSK modulated system with two transmit and two receive antennas, $E\left\{\left|x_{1}\right|^{2}\right\}=7 / 4$ and $E\left\{\left|x_{2}\right|^{2}\right\}=1 / 4$.

The average SEP of $x_{1}$ and $x_{2}$ are the solutions for equations (22). The analytical SEPs of $x_{1}$ and $x_{2}$ are compared with the simulation ones, as shown in Fig. 2 and Fig. 3 for $N_{r}=2$ and $N_{r}=4$, respectively. Clearly, analytical results match well with simulation results in the considered SNR region. These results demonstrate the accuracy of the proposed method under the situations of equal power allocation as well as unequal power allocation. This provides system designers with an effective method to predict the system performance, even when the SNR is low. Note that in most existing methods [3-8], there is a significant gap between the analytical and simulation results in low SNR situations.

\section{CONClusion}

This letter has presented a new analytical method to SEP analysis for ML detection in MIMO multiplexing systems. The SEP equations have been generated after deriving the closed-form conditional SEPs and the probabilities of the error events. The average SEP of each transmitted signal has been obtained by solving a set of SEP equations. The letter has also shown how SEPs can be obtained under imperfect channel

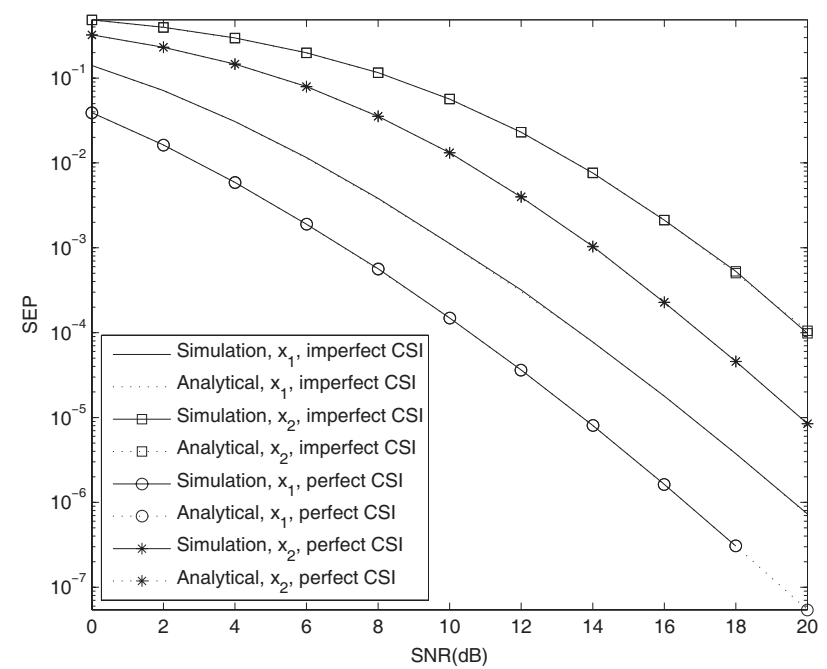

Fig. 3. SEP for a QPSK modulated system with two transmit and four receive antennas, $E\left\{\left|x_{1}\right|^{2}\right\}=7 / 4$ and $E\left\{\left|x_{2}\right|^{2}\right\}=1 / 4$.

estimation and unequal power allocation, in which the existing methods have difficulties in obtaining a solution. Monte-Carlo simulations have demonstrated that the proposed method can yield accurate results, even under low SNR.

\section{REFERENCES}

[1] D. Gesbert, M. Shafi, D. S. Shiu, P. J. Smith, and A. Naguib, "From theory to practice: an overview of MIMO space-time coded wireless systems," IEEE J. Select. Areas Commun., vol. 10, pp. 281-302, Apr. 2003.

[2] J. M. Wang and B. Daneshrad, "A comparative study of MIMO detection algorithms for wideband spatial multiplexing systems," in Proc. IEEE Conf. Wireless Commun. Networking, vol. 1, pp. 408-413, Mar. 2005.

[3] R. V. Nee, A. V. Zelst, and G. Awater, "Maximum likelihood decoding in a space division multiplexing system," in Proc. IEEE Veh. Technol. Conf., vol. 1, pp. 6-10, May 2000.

[4] M. Shin, D. S. Kwon, and C. Y. Lee, "Performance analysis of maximum likelihood detection for MIMO systems," in Proc. IEEE 63rd Veh. Technol. Conf., vol. 5, pp. 2154-2158, May 2006.

[5] G. Lindell, "Some exact union bound results for maximum likelihood detection in MIMO systems," in Proc. IEEE International Symp. Inform. Theory, pp. 2223-2227, Sept. 2005.

[6] S. J. Grant and J. K. Cavers, "Performance enhancement through joint detection of co-channel signals using diversity arrays," IEEE Trans. Commun., vol. 46, pp. 1038-1049, Aug. 1998.

[7] S. J. Grant and J. K. Cavers, "Further analytic results on the joint detection of co-channel signals using diversity arrays," IEEE Trans. Commun., vol. 48, pp. 1788-1792, Nov. 2000.

[8] X. Zhu and R. D. Murch, "Performance analysis of maximum likelihood detection in a MIMO antenna system," IEEE Trans. Commun., vol. 50, pp. 187-191, Feb. 2002.

[9] W. Y. Li and N. C. Beaulieu, "Effects of channel-estimation errors on receiver selection-combining schemes for Alamouti MIMO systems with BPSK," IEEE Trans. Commun., vol. 54, pp. 169-178, Jan. 2006.

[10] J. G. Proakis, Digital Communications. New York: McGraw Hill, 4th ed., 2001.

[11] G. L. Stuber, Principles of Mobile Communication. Kluwer Academic Publishers, 2000.

[12] J. W. Craig, "A new, simple and exact result for calculating the probability of error for two-dimensional signal constellations," in Proc. IEEE Military Commun. Conf., pp. 571-575, Nov. 1991.

[13] I. N. Bronshtein, K. A. Semendyayev, G. Musiol, and H. Muehlig, Handbook of Mathematics. Springer, 1997.

[14] A. Annamalai and C. Tellambura, "Error rates for Nakagami-m fading multichannel reception of binary and M-ary signals," IEEE Trans. Commun., vol. 49, pp. 58-68, Jan. 2001.

[15] M. Kay Steven, Fundamentals of Statistical Signal Processing. New York: Prentice Hall, 1993. 